QUANTUM DYNAMICS

BANACH CENTER PUBLICATIONS, VOLUME 120
INSTITUTE OF MATHEMATICS
POLISH ACADEMY OF SCIENCES
WARSZAWA 2020

\title{
A NOTE ON THE QUANTUM FAMILY OF MAPS
}

\author{
ALBERT JEU-LIANG SHEU \\ Department of Mathematics, University of Kansas \\ Lawrence, KS 66045, U.S.A. \\ ORCID:0000-0001-5613-0240_E-mail: asheu@ku.edu \\ THOMAS TIMMERMANN \\ FB Mathematik und Informatik, University of Münster \\ Münster, Germany \\ E-mail: timmermt@math.uni-muenster.de
}

\begin{abstract}
The notion and theory of the quantum space of all maps from a quantum space pioneered by Sołtan have been mainly focused on finite-dimensional $\mathrm{C}^{*}$-algebras which are matrix algebra bundles over a finite set $S$. We propose a modification of this notion to cover the case of $C(X)$ for general compact Hausdorff spaces $X$ instead of finite sets $S$ while taking into account of the topology of $X$. A notion of free product of copies of a unital $\mathrm{C}^{*}$-algebra topologically indexed by a compact Hausdorff space arises naturally, and satisfies some desired functoriality.
\end{abstract}

1. Introduction. In a series of papers [5, 6, 7], Soltan pioneered in the development and study of a theory of quantum spaces of maps between quantum spaces. Following a natural categorical viewpoint, he proposes a conceptual definition of the quantum space $\mathbb{A}$ of all maps from a quantum space $\mathbb{X}$ to a quantum space $\mathbb{Y}$, via the universality that there is a $\mathrm{C}^{*}$-algebra homomorphism $\eta: C(\mathbb{Y}) \rightarrow C(\mathbb{X}) \otimes C(\mathbb{A})$ such that for any quantum space $\mathbb{M}$ with a $\mathrm{C}^{*}$-algebra homomorphism $\psi: C(\mathbb{Y}) \rightarrow C(\mathbb{X}) \otimes C(\mathbb{M})$ (viewed as a quantum family of maps from $\mathbb{X}$ to $\mathbb{Y}$ parametrized by $\mathbb{M})$, there is a unique $\mathrm{C}^{*}$-algebra homomorphism

2010 Mathematics Subject Classification: 46L85.

Key words and phrases: quantum family of maps, free product of $\mathrm{C}^{*}$-algebras, relaxed tensor product.

This work was partially supported by the grant H2020-MSCA-RISE-2015-691246-QUANTUM DYNAMICS and the Polish government grant 3542/H2020/2016/2.

The paper is in final form and no version of it will be published elsewhere. 
$\bar{\psi}: C(\mathbb{A}) \rightarrow C(\mathbb{M})$ such that $\psi=(\mathrm{id} \otimes \bar{\psi}) \circ \eta$. Here we adopt the view that all $\mathrm{C}^{*}$-algebras $\mathcal{B}$ are the function algebra $C(\mathbb{Y})$ of some virtual quantum space $\mathbb{Y}$, and as Soltan does, we limit our consideration to unital $\mathrm{C}^{*}$-algebras or equivalently compact quantum spaces only. Most of Soltan's results are focused on finite-dimensional $\mathrm{C}^{*}$-algebras $C(\mathbb{X})$ and finitely generated $\mathrm{C}^{*}$-algebras $C(\mathbb{Y})$, since the existence of $\mathbb{A}$ is generally established only for such cases.

An attempt to extend the study of quantum spaces of all maps to cover infinitedimensional $\mathrm{C}^{*}$-algebras $C(\mathbb{X})$ was initiated in Kang's thesis [2], which considers the commutative $\mathrm{C}^{*}$-algebra $C(\mathbb{X}) \equiv C\left(\mathbb{N}^{+}\right)$for the one-point compactification $\mathbb{N}^{+}=\mathbb{N} \cup\{\infty\}$ of the discrete infinite space $\mathbb{N}$ of all natural numbers. It is noted that the quantum space $\mathbb{A}$ satisfying the above definition fails to exist since the operator-norm continuity required by the $\mathrm{C}^{*}$-algebra tensor product $C\left(\mathbb{N}^{+}\right) \otimes \mathcal{B}(\mathcal{H}) \cong C\left(\mathbb{N}^{+}, \mathcal{B}(\mathcal{H})\right)$ does not survive under taking an infinite direct sum $\oplus$ of operators.

In this short note, we report some observations made in extending the approach taken in Kang's thesis to propose a version of the quantum space of all maps from a classical compact Hausdorff space $X$, i.e. the quantum space underlying a unital commutative $\mathrm{C}^{*}$-algebra $C(\mathbb{X}) \equiv C(X)$, to a compact quantum space $\mathbb{Y}$.

This work was carried out during the first author's visit to the second author at the University of Münster in April of 2018. The first author would like to thank the second author and the Mathematics Department of the University of Münster for their warm accommodation, hospitality, and support during his visit.

2. Relaxed $\mathbf{C}^{*}$-algebras. In order to cover the case of $C(\mathbb{X})=C\left(\mathbb{N}^{+}\right)$for the compact infinite topological space $\mathbb{N}^{+} \equiv \mathbb{N} \cup\{\infty\}$, the ordinary notion of (minimal) $\mathrm{C}^{*}$-algebra tensor product $C\left(\mathbb{N}^{+}\right) \otimes \mathcal{B}$ was modified in Kang's thesis [2]. A key idea in the approach taken was to replace norm continuity in the notion $C\left(\mathbb{N}^{+}\right) \otimes \mathcal{B}$ by strong continuity in a representation theoretic context.

In this paper, to handle compact Hausdorff spaces in full generality, we will start with replacing the minimal tensor product functor $C(X) \otimes-$ by a "relaxed" variant $C(X) \otimes-$ whose definition involves an additional locally convex topology on the second factor.

In the following discussion, unless otherwise stated, *-algebras, *-homomorphisms,

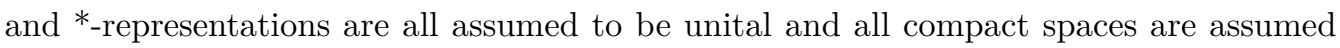
to be Hausdorff.

Definition 1. A relaxed $C^{*}$-algebra is a $\mathrm{C}^{*}$-algebra $\mathcal{A}$ with an additional locally convex topology $\mathfrak{T}$, called the "relaxed topology" of $\mathcal{A}$, which is determined by a separating family $S$ of norm-continuous seminorms on $\mathcal{A}$, making each of the multiplication, involution and addition operations of $\mathcal{A}$ continuous on the open unit ball $(\mathcal{A})_{1}$ of $\mathcal{A}$ (and hence on any bounded subset of $\mathcal{A}$ ), e.g. the multiplication binary operation

$$
(\mathcal{A})_{1} \times(\mathcal{A})_{1} \ni(a, b) \mapsto a b \in(\mathcal{A})_{1}
$$

is (jointly) continuous when each copy of $(\mathcal{A})_{1}$ involved is equipped with the inherited topology $\mathfrak{T}$. 
Definition 2. A morphism of unital relaxed $\mathrm{C}^{*}$-algebras, also called a relaxed *-homomorphism, is a unital *-homomorphism that is continuous with respect to the relaxed topologies.

We denote by $\mathfrak{r C}^{*}$ the category of all unital relaxed $\mathrm{C}^{*}$-algebras.

ExAMPLE. Let $\mathcal{A}$ be a unital $\mathrm{C}^{*}$-algebra with a faithful representation $\pi$ on some Hilbert space $\mathcal{H}$, and denote by $\mathfrak{T}_{\pi}$ the topology on $\mathcal{A}$ pulled back via $\pi$ from the ${ }^{*}$-strong topology on $\mathcal{B}(\mathcal{H})$. Then $\left(\mathcal{A}, \mathfrak{T}_{\pi}\right)$ is a unital relaxed $\mathrm{C}^{*}$-algebra.

In particular, given a Hilbert space $\mathcal{H}$, all unital $\mathrm{C}^{*}$-algebras $\mathcal{A} \subseteq \mathcal{B}(\mathcal{H})$ can be regarded as relaxed $\mathrm{C}^{*}$-algebras in a natural way. Thus, all unital $\mathrm{C}^{*}$-subalgebras of $\mathcal{B}(\mathcal{H})$ yield a full subcategory $\mathfrak{r C}_{\mathcal{H}}^{*}$ of $\mathfrak{r C}^{*}$. Here we remark that it might be of interest to focus on specific full subcategories like this one, for example, the construction of a topological free product $\mathcal{B}^{\circledast X}$ discussed later in this paper could be carried out in this subcategory $\mathfrak{r C}_{\mathcal{H}}^{*}$, which would potentially give us another $\mathrm{C}^{*}$-algebra.

Denote by $\mathfrak{C}^{*}$ the category formed by all unital $\mathrm{C}^{*}$-algebras and all unital *-homomorphisms.

3. The relaxed tensor product. Let $X$ be a compact Hausdorff space and $(\mathcal{A}, \mathfrak{T})$ be a unital relaxed $\mathrm{C}^{*}$-algebra. We denote by

$$
C(X) \otimes(\mathcal{A}, \mathfrak{T})
$$

the set of all functions $f: X \rightarrow \mathcal{A}$ that are norm-bounded and continuous with respect to the relaxed topology $\mathfrak{T}$ on $\mathcal{A}$.

LEMma 1. The set $C(X) \otimes(\mathcal{A}, \mathfrak{T})$ is a $C^{*}$-algebra with respect to the point-wise operations and the supremum norm.

Proof. By the assumption on $\mathfrak{T}$, the set $C(X) \otimes(\mathcal{A}, \mathfrak{T})$ is closed under point-wise addition, multiplication and involution of functions. Let $\left(f_{n}\right)_{n}$ be a sequence in $C(X) \otimes(\mathcal{A}, \mathfrak{T})$ converging to some function $f: X \rightarrow \mathcal{A}$ with respect to the supremum norm. We need to show that $f$ is continuous with respect to the relaxed topology on $\mathcal{A}$. Recall that $\mathfrak{T}$ is determined by some separating family $S$ of norm-continuous seminorms on $\mathcal{A}$. Given any semi-norm $\rho \in S$, a point $x \in X$ and an $\epsilon>0$, we can choose

1. $C>0$ such that $\rho \leq C\|-\|$,

2. $N$ such that $\left\|f_{n}-f\right\|_{\infty} \leq \epsilon /(3 C)$ for all $n>N$, and

3. a neighbourhood $U$ of $x$ such that $\rho\left(f_{n}(y)-f_{n}(x)\right)<\epsilon / 3$ for all $y \in U$.

Then for all $y \in U$,

$$
\begin{aligned}
\rho(f(y)-f(x)) & \leq \rho\left(f(y)-f_{n}(y)\right)+\rho\left(f_{n}(y)-f_{n}(x)\right)+\rho\left(f_{n}(x)-f(x)\right) \\
& \leq C \cdot \epsilon /(3 C)+\epsilon / 3+C \cdot \epsilon /(3 C)=\epsilon .
\end{aligned}
$$

So $f$ is continuous with respect to the topology determined by any $\rho \in S$, and hence continuous with respect to the topology $\mathfrak{T}$ determined by $S$.

Definition 3. Let $X$ be a compact Hausdorff space and let $(\mathcal{A}, \mathfrak{T})$ be a unital relaxed $\mathrm{C}^{*}$-algebra. Then the relaxed tensor product of $C(X)$ and $(\mathcal{A}, \mathfrak{T})$ is the $\mathrm{C}^{*}$-algebra $C(X) \otimes(\mathcal{A}, \mathfrak{T})$. 
By construction, the relaxed tensor product is functorial in the following sense:

Lemma 2. Given a continuous map $F: X \rightarrow Y$ of compact Hausdorff spaces and a morphism $\pi$ of relaxed $C^{*}$-algebras $\left(\mathcal{A}, \mathfrak{T}_{\mathcal{A}}\right)$ and $\left(\mathcal{B}, \mathfrak{T}_{\mathcal{B}}\right)$, there exists a unital ${ }^{*}$-homomorphism

$$
F^{*} \otimes \pi: C(Y) \otimes\left(\mathcal{A}, \mathfrak{T}_{\mathcal{A}}\right) \ni f \mapsto \pi \circ f \circ F \in C(X) \otimes\left(\mathcal{B}, \mathfrak{T}_{\mathcal{B}}\right) .
$$

The proof is trivial.

4. Quantum family of maps. Now we give a modified definition of the quantum space $\mathbb{A}$ of all maps from a compact Hausdorff space $X$ to a compact quantum space $\mathbb{Y}$.

Conceptually we will replace the notion of a quantum space $\mathbb{M}$ of maps or its $\mathrm{C}^{*}$-algebra $C(\mathbb{M})$ by a relaxed unital $\mathrm{C}^{*}$-algebra $\left(C(\mathbb{M}), \mathfrak{T}_{\mathbb{M}}\right)$, and replace the ordinary (minimal) tensor product $\otimes$ by the relaxed tensor product $\otimes$.

Definition 4. The quantum space $\mathbb{A}$ of all maps from a compact Hausdorff space $X$ to a compact quantum space $\mathbb{Y}$ is the relaxed $\mathrm{C}^{*}$-algebra $\left(C(\mathbb{A}), \mathfrak{T}_{\mathbb{A}}\right)$ (if exists) with a unital $\mathrm{C}^{*}$-algebra homomorphism $\eta: C(\mathbb{Y}) \rightarrow C(X) \otimes\left(C(\mathbb{A}), \mathfrak{T}_{\mathbb{A}}\right)$ such that for any unital relaxed $\mathrm{C}^{*}$-algebra $\left(C(\mathbb{M}), \mathfrak{T}_{\mathbb{M}}\right)$ with a unital ${ }^{*}$-homomorphism $\psi: C(\mathbb{Y}) \rightarrow$ $C(X) \otimes\left(C(\mathbb{M}), \mathfrak{T}_{\mathbb{M}}\right)$, there is a unique unital relaxed ${ }^{*}$-homomorphism $\bar{\psi}:\left(C(\mathbb{A}), \mathfrak{T}_{\mathbb{A}}\right) \rightarrow$ $\left(C(\mathbb{M}), \mathfrak{T}_{\mathbb{M}}\right)$ such that $\psi=($ id $\otimes \bar{\psi}) \circ \eta$. We call a unital *-homomorphism $\phi: C(\mathbb{Y}) \rightarrow$ $C(X) \otimes\left(C(\mathbb{M}), \mathfrak{T}_{\mathbb{M}}\right)$ a quantum family of maps from $X$ to $\mathbb{Y}$ parametrized by $\mathbb{M}$.

Our aim is to show that such a quantum space $\mathbb{A}$ exists in general. In fact, a suitably defined free product $\mathcal{B}^{\circledast X}$ of copies of $\mathcal{B} \equiv C(\mathbb{Y})$ topologically parametrized by $X$ is shown in the next section to be $C(\mathbb{A})$, generalizing Soltan's and Kang's results on the existence of $\mathbb{A}$ for $X$ finite or $X=\mathbb{N}^{+}$.

5. The universal quantum family of maps. Given a compact Hausdorff space $X$ and a unital $\mathrm{C}^{*}$-algebra $\mathcal{B}$, we denote by $\mathcal{B}^{* X}$ the unital free product of $|X|$ copies of $\mathcal{B}$ and by $\iota_{x, \mathcal{B}}: \mathcal{B} \rightarrow \mathcal{B}^{* X}$ the canonical $\mathrm{C}^{*}$-algebra embedding associated to each point $x \in X$, where $|X|$ is the cardinality of $X$. The assignment $\mathcal{B} \mapsto \mathcal{B}^{* X}$ extends to a functor as follows. Given a map $F: X \rightarrow Y$ of compact Hausdorff spaces and a unital *-homomorphism $\pi: \mathcal{B} \rightarrow \mathcal{C}$, we obtain a unital *-homomorphism

$$
\pi^{* F}: \mathcal{B}^{* X} \ni \iota_{x, \mathcal{B}}(b) \mapsto \iota_{F(x), \mathcal{C}}(\pi(b)) \in \mathcal{C}^{* Y} \quad \text { for } x \in X \text { and } b \in \mathcal{B} .
$$

Now, the assignments $(X, \mathcal{B}) \mapsto \mathcal{B}^{* X}$ and $(F, \pi) \mapsto \pi^{* F}$ form a functor from $\mathfrak{C o m p} \times \mathfrak{C}^{*}$ to $\mathfrak{C}^{*}$, where $\mathfrak{C} \mathfrak{m} \mathfrak{p}$ denotes the category of compact Hausdorff spaces with continuous maps, and fixing $X$, we obtain a functor $(-)^{* X}$ on $\mathfrak{C}^{*}$.

Clearly this notion of free product $\mathcal{B}^{* X}$ works for any set $X$, and existing studies of free products (e.g. [1] for a reduced version and [8]) mainly focused on a finite set $X$ while showing important connections to quantum theory. Now bringing the topology of $X$ into consideration, we define a smaller but more topologically sensitive free product $\mathrm{C}^{*}$-algebra $\mathcal{B}^{\circledast X}$. 
Let $\mathcal{B}$ be a unital $\mathrm{C}^{*}$-algebra again. Given a unital relaxed $\mathrm{C}^{*}$-algebra $(\mathcal{A}, \mathfrak{T})$, we call a unital *-homomorphism $\pi: \mathcal{B}^{* X} \rightarrow \mathcal{A}$ admissible if for every $b \in \mathcal{B}$, the map

$$
X \ni x \mapsto \pi\left(\iota_{x, \mathcal{B}}(b)\right) \in \mathcal{A}
$$

is continuous with respect to the relaxed topology $\mathfrak{T}$ on $\mathcal{A}$. Let

$$
J_{X, \mathcal{B}}:=\bigcap_{\pi} \operatorname{ker} \pi \subseteq \mathcal{B}^{* X},
$$

where the intersection is taken over all unital relaxed $\mathrm{C}^{*}$-algebras $(\mathcal{A}, \mathfrak{T})$ and all admissible *-homomorphisms $\pi$ from $\mathcal{B}^{* X}$ to the underlying $\mathrm{C}^{*}$-algebra $\mathcal{A}$. Denote by

$$
\mathcal{B}^{\circledast X}:=\mathcal{B}^{* X} / J_{X, \mathcal{B}}
$$

the quotient $\mathrm{C}^{*}$-algebra, by

$$
\eta_{X, \mathcal{B}}: \mathcal{B}^{* X} \rightarrow \mathcal{B}^{\circledast X}
$$

the quotient map and let

$$
\bar{\iota}_{x, \mathcal{B}}:=\eta_{X, \mathcal{B}} \circ \iota_{x, \mathcal{B}}: \mathcal{B} \rightarrow \mathcal{B}^{\circledast X}
$$

for every $x \in X$.

Denote by $\mathfrak{T}_{\mathcal{B}^{\circledast X} X}$ the weakest topology on $\mathcal{B}^{\circledast X}$ that makes $\pi^{\prime}: \mathcal{B}^{\circledast X} \rightarrow(\mathcal{A}, \mathfrak{T})$ continuous with respect to the relaxed topology $\mathfrak{T}$ on $\mathcal{A}$ for any admissible ${ }^{*}$-homomorphism $\pi: \mathcal{B}^{* X} \rightarrow(\mathcal{A}, \mathfrak{T})$, where $\pi^{\prime}$ is the unique ${ }^{*}$-homomorphism determined by $\pi^{\prime} \circ \eta_{X, \mathcal{B}}=\pi$. Then $\left(\mathcal{B}^{\circledast X}, \mathfrak{T}_{\mathcal{B}^{\circledast X}}\right)$ evidently is a relaxed $\mathrm{C}^{*}$-algebra with $\mathfrak{T}_{\mathcal{B}^{\circledast} X}$ determined by the set $S_{\mathcal{B} \circledast X}$ consisting of seminorms $\bar{\rho}$ on $\mathcal{B}^{\circledast X}$ such that $\bar{\rho} \circ \eta_{X, \mathcal{B}}=\rho \circ \pi$ for some admissible *-homomorphism $\pi: \mathcal{B}^{* X} \rightarrow(\mathcal{A}, \mathfrak{T})$ and some $\rho$ in the set $S$ of seminorms on $\mathcal{A}$ that determines the topology $\mathfrak{T}$.

Proposition 1. Let $\mathcal{B}$ be a unital $C^{*}$-algebra. Then there exists a unital *-homomorphism

$$
\alpha_{\mathcal{B}}: \mathcal{B} \rightarrow C(X) \otimes\left(\mathcal{B}^{\circledast X}, \mathfrak{T}_{\mathcal{B} \circledast X}\right)
$$

such that for all $b \in \mathcal{B}$ and $x \in X$,

$$
\alpha_{\mathcal{B}}(b)(x)=\bar{\iota}_{x, \mathcal{B}}(b)=\left(\eta_{X, \mathcal{B}} \circ \iota_{x, \mathcal{B}}\right)(b),
$$

or equivalently, $\eta_{X, \mathcal{B}}: \mathcal{B}^{* X} \rightarrow \mathcal{B}^{\circledast X}$ is an admissible ${ }^{*}$-homomorphism.

Proof. We only need to check that for every $b \in \mathcal{B}$, the map $\alpha_{\mathcal{B}}(b): X \rightarrow \mathcal{B}^{\circledast X}$ is continuous with respect to the relaxed topology. So, we take a semi-norm $\bar{\rho}$ that arises by factorizing a composition $\rho \circ \pi$ as above, that is, $\bar{\rho} \circ \eta_{X, \mathcal{B}}=\rho \circ \pi$. Then the map

$$
x \mapsto \bar{\rho}\left(\bar{\iota}_{x, \mathcal{B}}(b)\right)=\left(\rho \circ \pi \circ \iota_{x, \mathcal{B}}\right)(b)
$$

is continuous because $\pi$ is admissible.

Clearly, the assignment

$$
(X, \mathcal{B}) \mapsto\left(\mathcal{B}^{\circledast X}, \mathfrak{T}_{\mathcal{B} \circledast X}\right)
$$

extends to a functor

$$
(-)^{\circledast(-)}: \mathfrak{C o m p} \times \mathfrak{C}^{*} \rightarrow \mathfrak{r} \mathfrak{C}^{*} .
$$

THEOREM 1. Let $X$ be a compact Hausdorff space. Then the functor $(-)^{\circledast X}: \mathfrak{C}^{*} \rightarrow \mathfrak{r C}^{*}$ is left adjoint to the functor $C(X) \otimes-: \mathfrak{r} \mathfrak{C}^{*} \rightarrow \mathfrak{C}^{*}$. 
Proof. Let $\mathcal{B}$ be a unital $\mathrm{C}^{*}$-algebra and $(\mathcal{A}, \mathfrak{T})$ a unital relaxed $\mathrm{C}^{*}$-algebra with $\mathfrak{T}$ determined by a separating family $S$ of norm-continuous seminorms on $\mathcal{A}$.

We claim that the map

$$
\operatorname{hom}_{\mathfrak{r} \mathfrak{C}^{*}}\left(\left(\mathcal{B}^{\circledast X}, \mathfrak{T}_{\mathcal{B}^{\circledast}}\right),(\mathcal{A}, \mathfrak{T})\right) \ni \phi \mapsto \tilde{\phi}:=(\operatorname{id} \otimes \phi) \circ \alpha_{\mathcal{B}} \in \operatorname{hom}_{\mathfrak{C}^{*}}(\mathcal{B}, C(X) \otimes(\mathcal{A}, \mathfrak{T}))
$$

is bijective. Indeed, given a unital *-homomorphism $\psi: \mathcal{B} \rightarrow C(X) \otimes(\mathcal{A}, \mathfrak{T})$, the unital *-homomorphism $\Psi: \mathcal{B}^{* X} \rightarrow \mathcal{A}$ defined by $\Psi\left(\iota_{x, \mathcal{B}}(b)\right):=\psi(b)(x)$ is admissible and therefore factorizes to a ${ }^{*}$-homomorphism $\bar{\psi}: \mathcal{B}^{\circledast X} \rightarrow \mathcal{A}$ such that $\bar{\psi} \circ \eta_{X, \mathcal{B}}=\Psi$. This *-homomorphism $\bar{\psi}$ is relaxed because $\mathfrak{T}_{\mathcal{B}^{\circledast X}}$ is defined as the weakest topology on $\mathcal{B}^{\circledast X}$ that makes $\pi^{\prime}$ continuous for all admissible ${ }^{*}$-homomorphisms $\pi$, and $\pi^{\prime}=\bar{\psi}$ when $\pi=\Psi$. Clearly, the assignment $\psi \mapsto \bar{\psi}$ is inverse to the assignment $\phi \mapsto \tilde{\phi}$.

Moreover, it is straightforward to check that both assignments $\psi \mapsto \bar{\psi}$ and $\phi \mapsto \tilde{\phi}$ are natural in $\mathcal{B}$ and $(\mathcal{A}, \mathfrak{T})$.

As a consequence of this categorical statement, we get the following existence theorem for the quantum space of all maps from a compact Hausdorff space $X$ to a compact quantum space.

THEOREM 2. The quantum space $\mathbb{A}$ of all maps from a compact Hausdorff space $X$ to a compact quantum space $\mathbb{Y}$ (or equivalently, a unital $C^{*}$-algebra $\mathcal{B} \equiv C(\mathbb{Y})$ ) exists and $\left(C(\mathbb{A}), \mathfrak{T}_{\mathbb{A}}\right)=\left(\mathcal{B}^{\circledast X}, \mathfrak{T}_{\mathcal{B}^{\circledast X}}\right)$ the topological free product of copies of $\mathcal{B}$ parametrized by $X$. More precisely, the ${ }^{*}$-homomorphism $\alpha_{\mathcal{B}}: \mathcal{B} \rightarrow C(X) \otimes\left(\mathcal{B}^{\circledast X}, \mathfrak{T}_{\mathcal{B} \circledast X}\right)$ satisfies the universality that for any unital $C^{*}$-algebra homomorphism $\psi: \mathcal{B} \rightarrow C(X) \otimes\left(C(\mathbb{M}), \mathfrak{T}_{\mathbb{M}}\right)$, there is a unique unital relaxed ${ }^{*}$-homomorphism $\bar{\psi}:\left(\mathcal{B}^{\circledast X}, \mathfrak{T}_{\mathcal{B} \circledast X}\right) \rightarrow\left(C(\mathbb{M}), \mathfrak{T}_{\mathbb{M}}\right)$ such that $\psi=(\mathrm{id} \otimes \bar{\psi}) \circ \alpha_{\mathcal{B}}$.

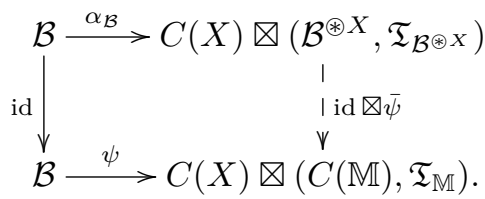

Proof. The assignment $\psi \mapsto \bar{\psi}$ constructed in the proof of Theorem 1 satisfies the stated universality exactly.

Since for a finite set $N$, the free product $\mathcal{B}^{*|N|} \equiv \mathcal{B}^{* N}$ of $|N|$ copies of a $\mathrm{C}^{*}$-algebra $\mathcal{B}$ is characterized by the universality condition in Soltan's definition of quantum space of all maps from $N$ to $\mathcal{B}$, the above theorem motivates our definition of the topological free product $\mathcal{B}^{\circledast X}$ of a family of copies of $\mathcal{B}$ parametrized by a topological space $X$. As a special case, the theorem says that given any collection of ${ }^{*}$-representations $\psi_{x}: \mathcal{B} \rightarrow \mathcal{B}(\mathcal{H})$ parametrized by $x \in X$ such that for all $b \in \mathcal{B}, x \mapsto \psi_{x}(b)$ is strongly continuous (and hence ${ }^{*}$-strongly continuous since $\mathcal{B}$ is involutive) on $X$, there is a unique *-representation $\bar{\psi}: \mathcal{B}^{\circledast X} \rightarrow \mathcal{B}(\mathcal{H})$ such that $\bar{\psi} \circ \bar{\iota}_{x, \mathcal{B}}=\psi_{x}$ for all $x \in X$.

It is easy to see that $\mathcal{B}^{* N}=\mathcal{B}^{\circledast N}$ when $N$ is a finite (discrete) space. But in general, it is not clear whether $\mathcal{B}^{* N} \equiv \mathcal{B}^{\circledast N}$ is embedded in $\mathcal{B}^{\circledast X}$ for any finite subset $N$ of a compact Hausdorff space $X$. It seems that a non-discrete topology on $X$ can seriously limit the existence of enough many admissible ${ }^{*}$-representations of $\mathcal{B}^{* X}$. However $\mathcal{B}^{* N}$ is embedded 
in $\mathcal{B}^{\circledast X}$ for any finite open subset $N$ of $X$ since any ${ }^{*}$-representation $\rho: \mathcal{B}^{* N} \rightarrow \mathcal{B}(\mathcal{H})$ can be extended to a ${ }^{*}$-representation $\pi$ of $\mathcal{B}^{* X}$ with $x \mapsto \pi\left(\iota_{x, \mathcal{B}}(b)\right){ }^{*}$-strongly continuous on $X$ by setting $\pi\left(\iota_{x, \mathcal{B}}(b)\right):=\rho\left(\iota_{x, \mathcal{B}}(b)\right) \otimes I_{\mathcal{H}_{0}}$ for $x \in N$ and $\pi\left(\iota_{x, \mathcal{B}}(b)\right):=I_{\mathcal{H}} \otimes \pi_{0}(b)$ for all $x \in X \backslash N$, where $\pi_{0}: \mathcal{B} \rightarrow \mathcal{B}\left(\mathcal{H}_{0}\right)$ can be any fixed unital *-representation of $\mathcal{B}$.

6. Some functorial properties. In this section, we discuss some functorial properties about the notion of topological free product $\mathcal{B}^{\circledast X}$.

We recall that in the category of unital $\mathrm{C}^{*}$-algebras, the pushout for a pair of $\mathrm{C}^{*}$-algebra homomorphisms $\mathcal{C} \stackrel{h}{\rightarrow} \mathcal{A}$ and $\mathcal{C} \stackrel{k}{\rightarrow} \mathcal{B}$ is given by the amalgamated product $\mathcal{A} *_{\mathcal{C}} \mathcal{B}$. For the theory of amalgamated product of $\mathrm{C}^{*}$-algebras or pushout in the category of $\mathrm{C}^{*}$-algebras, we refer to the systematic study given by $\mathrm{G}$. Pedersen in [4].

Proposition 2. The functor $(-)^{\circledast X}$ preserves pushout in the sense that for any pushout $\mathcal{A} *_{\mathcal{C}} \mathcal{B}$ given by morphisms $\mathcal{C} \stackrel{h}{\rightarrow} \mathcal{A}$ and $\mathcal{C} \stackrel{k}{\rightarrow} \mathcal{B}$ in the category $\mathfrak{C}^{*}$, if $\left(\mathcal{A}^{\circledast X}, \mathfrak{T}_{\mathcal{A}}^{\circledast X}\right) \stackrel{H}{\rightarrow}$ $\left(\mathcal{D}, \mathfrak{T}_{\mathcal{D}}\right)$ and $\left(\mathcal{B}^{\circledast X}, \mathfrak{T}_{\mathcal{B} \circledast X}\right) \stackrel{K}{\rightarrow}\left(\mathcal{D}, \mathfrak{T}_{\mathcal{D}}\right)$ are relaxed ${ }^{*}$-homomorphisms such that $H \circ h^{\circledast X}=$ $K \circ k^{\circledast X}$, then there is a unique relaxed ${ }^{*}$-homomorphism $\Psi:\left(\left(\mathcal{A} *_{\mathcal{C}} \mathcal{B}\right)^{\circledast X}, \mathfrak{T}_{\left(\mathcal{A} *_{\mathcal{C}} \mathcal{B}\right) \circledast X}\right) \rightarrow$ $\left(\mathcal{D}, \mathfrak{T}_{\mathcal{D}}\right)$ such that $H=\Psi \circ\left(\iota_{\mathcal{A}}\right)^{\circledast X}$ and $K=\Psi \circ\left(\iota_{\mathcal{B}}\right)^{\circledast X}$, where $\iota_{\mathcal{A}}: \mathcal{A} \rightarrow \mathcal{A} *_{\mathcal{C}} \mathcal{B}$ and $\iota_{\mathcal{B}}: \mathcal{B} \rightarrow \mathcal{A} *_{\mathcal{C}} \mathcal{B}$ are the canonical ${ }^{*}$-homomorphisms.

Proof. Since left adjoint functors preserve colimits in the category theory ([3, section V.5, p. 115]) and the functor $(-)^{\circledast X}: \mathfrak{C}^{*} \rightarrow \mathfrak{r} \mathfrak{C}^{*}$ is shown in Theorem 1 to be left adjoint to the functor $C(X) \otimes-: \mathfrak{r} \mathfrak{C}^{*} \rightarrow \mathfrak{C}^{*}$, the assignment $\mathcal{B} \mapsto\left(\mathcal{B}^{\circledast X}, \mathfrak{T}_{\mathcal{B} \circledast}\right)$ preserves the colimits and in particular, the pushouts.

Now we consider another functor associated with the construction of topological free product $\mathcal{B}^{\circledast X}$.

Proposition 3. Given a $C^{*}$-algebra $\mathcal{B}$ and a continuous map $f: X \rightarrow Y$ between compact Hausdorff spaces $X$ and $Y$, there is a unique well-defined relaxed ${ }^{*}$-homomorphism $\mathcal{B}^{\circledast f}:\left(\mathcal{B}^{\circledast X}, \mathfrak{T}_{\mathcal{B} \circledast X}\right) \rightarrow\left(\mathcal{B}^{\circledast Y}, \mathfrak{T}_{\mathcal{B} \circledast Y}\right)$ such that $\mathcal{B}^{\circledast f}\left(\bar{\iota}_{x, \mathcal{B}}(b)\right)=\bar{\iota}_{f(x), \mathcal{B}}(b)$ for all $x \in X$ and $b \in \mathcal{B}$.

Proof. Clearly since $\bar{\iota}_{x, \mathcal{B}}(b)$ for $(x, b) \in X \times \mathcal{B}$ generate the $\mathrm{C}^{*}$-algebra $\mathcal{B}^{\circledast} X$, the uniqueness of $\mathcal{B}^{\circledast f}$ is clear. It remains to show that $\mathcal{B}^{\circledast f}$ can be well-defined as a relaxed *homomorphism.

Note that the map $f$ induces a well-defined *-homomorphism

$$
f^{*} \otimes \mathrm{id}: C(Y) \otimes\left(\mathcal{B}^{\circledast Y}, \mathfrak{T}_{\mathcal{B}^{\circledast Y}}\right) \ni g \mapsto g \circ f \in C(X) \otimes\left(\mathcal{B}^{\circledast Y}, \mathfrak{T}_{\mathcal{B}^{\circledast Y}}\right)
$$

because $g \circ f: X \rightarrow\left(\mathcal{B}^{\circledast Y}, \mathfrak{T}_{\mathcal{B} \circledast Y}\right)$ is continuous if $g: Y \rightarrow\left(\mathcal{B}^{\circledast Y}, \mathfrak{T}_{\mathcal{B} \circledast Y}\right)$ is, and clearly $\|g \circ f\|_{\infty} \leq\|g\|_{\infty}$.

Let $\alpha_{Y, \mathcal{B}}: \mathcal{B} \rightarrow C(Y) \otimes\left(\mathcal{B}^{\circledast Y}, \mathfrak{T}_{\mathcal{B} \circledast Y}\right)$ be the canonical *-homomorphism $\alpha_{\mathcal{B}}$ associated with the space $Y$ instead of $X$. By applying Theorem 2 to the ${ }^{*}$-homomorphism

$$
\psi:=\left(f^{*} \otimes \mathrm{id}\right) \circ \alpha_{Y, \mathcal{B}}: \mathcal{B} \rightarrow C(X) \otimes\left(\mathcal{B}^{\circledast Y}, \mathfrak{T}_{\mathcal{B} \circledast Y}\right)
$$


shown in the diagram

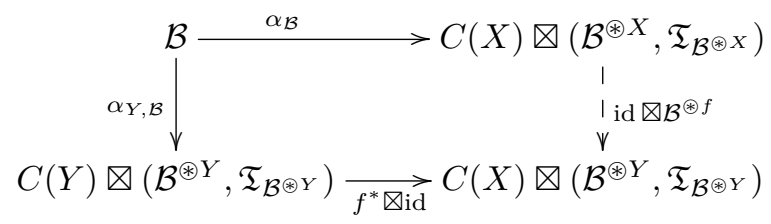

we get a unique relaxed *-homomorphism denoted as $\mathcal{B}^{\circledast f}:\left(\mathcal{B}^{\circledast X}, \mathfrak{T}_{\mathcal{B} \circledast X}\right) \rightarrow\left(\mathcal{B}^{\circledast Y}, \mathfrak{T}_{\mathcal{B} \circledast Y}\right)$ making this diagram commute. Now for all $(x, b) \in X \times \mathcal{B}$,

$$
\begin{aligned}
\mathcal{B}^{\circledast f}\left(\bar{\iota}_{x, \mathcal{B}}(b)\right) & =\mathcal{B}^{\circledast f}\left(\alpha_{\mathcal{B}}(b)(x)\right)=\left(\left(\left(f^{*} \otimes \mathrm{id}\right) \circ \alpha_{Y, \mathcal{B}}\right)(b)\right)(x) \\
& =\left(\left(f^{*} \otimes \mathrm{id}\right)\left(\alpha_{Y, \mathcal{B}}(b)\right)\right)(x)=\left(\alpha_{Y, \mathcal{B}}(b)\right)(f(x))=\bar{\iota}_{f(x), \mathcal{B}}(b)
\end{aligned}
$$

where it is understood that $\bar{\iota}_{x, \mathcal{B}}: \mathcal{B} \rightarrow \mathcal{B}^{\circledast X}$ and $\bar{\iota}_{f(x), \mathcal{B}}: \mathcal{B} \rightarrow \mathcal{B}^{\circledast Y}$ are embeddings of $\mathcal{B}$ into different free products.

COROLlary 1. The assignment $X \mapsto\left(\mathcal{B}^{\circledast X}, \mathfrak{T}_{\mathcal{B}^{\circledast X}}\right)$ and $f \mapsto \mathcal{B}^{\circledast f}$ for continuous maps $f: X \rightarrow Y$ constitute a covariant functor $\mathcal{B}^{\circledast-}: \mathfrak{C o m p}^{*} \rightarrow \mathfrak{r C}^{*}$ from the category of compact Hausdorff spaces to the category of unital relaxed $C^{*}$-algebras, for any given unital $C^{*}$-algebra $\mathcal{B}$.

Proof. For any $X \stackrel{f}{\rightarrow} Y \stackrel{g}{\rightarrow} Z, x \in X$, and $b \in \mathcal{B}$,

$$
\begin{aligned}
\mathcal{B}^{\circledast(g \circ f)}\left(\bar{\iota}_{x, \mathcal{B}}(b)\right) & =\bar{\iota}_{(g \circ f)(x), \mathcal{B}}(b)=\bar{\iota}_{g(f(x)), \mathcal{B}}(b) \\
& =\mathcal{B}^{\circledast g}\left(\bar{\iota}_{f(x), \mathcal{B}}(b)\right)=\mathcal{B}^{\circledast g}\left(\mathcal{B}^{\circledast f}\left(\bar{\iota}_{x, \mathcal{B}}(b)\right)\right)
\end{aligned}
$$

implies that $\mathcal{B}^{\circledast(g \circ f)}=\mathcal{B}^{\circledast g} \circ \mathcal{B}^{\circledast f}$.

Given continuous maps $Z \stackrel{f}{\rightarrow} X$ and $Z \stackrel{g}{\rightarrow} Y$ between compact Hausdorff spaces, the pushout $X \cup_{Z} Y$ in the category of compact Hausdorff spaces for the pair $(f, g)$ is well defined and can be constructed as follows.

Let $\mathfrak{R}$ be the collection of all equivalence relations $R \subset(X \sqcup Y) \times(X \sqcup Y)$ on $X \sqcup Y$ such that $(f(z), g(z)) \in R$ for all $z \in Z$ and the (automatically compact) quotient topological space $(X \sqcup Y) / R$ is Hausdorff. Clearly $\mathfrak{R}$ is not empty since it contains the equivalence relation $(X \sqcup Y) \times(X \sqcup Y)$. It is clearly that the intersection $\sim$ of all $R \in \mathfrak{R}$ is an equivalence relation on $X \sqcup Y$ containing $(f(z), g(z))$ for all $z \in Z$. Furthermore the quotient space $(X \sqcup Y) / \sim$ is still Hausdorff. In fact, if $[p] \neq[q]$ in $(X \sqcup Y) / \sim$ for some $p, q \in X \sqcup Y$, then $(p, q) \notin \sim$ and hence $(p, q) \notin R$ for some $R \in \mathfrak{R}$, which implies that the two distinct points $[p]_{R}$ and $[q]_{R}$ in the Hausdorff space $(X \sqcup Y) / R$ are separated by disjoint open sets $U$ and $V$ of $(X \sqcup Y) / R$. Now the inverse images of $U$ and $V$ under the canonical quotient map $(X \sqcup Y) / \sim \rightarrow(X \sqcup Y) / R$ are disjoint open sets separating $[p]$ and $[q]$ in $(X \sqcup Y) / \sim$. So $(X \sqcup Y) / \sim$ is Hausdorff.

It is routine to check that the compact Hausdorff space $X \cup_{Z} Y:=(X \sqcup Y) / \sim$ is a pushout for the pair $(f, g)$, i.e. for any continuous maps $f^{\prime}: X \rightarrow W$ and $g^{\prime}: Y \rightarrow W$ with $f^{\prime} \circ f=g^{\prime} \circ g$, there is a unique continuous map $h: X \cup_{Z} Y \rightarrow W$ such that $h \circ \varepsilon_{X}=f^{\prime}$ and $h \circ \varepsilon_{Y}=g^{\prime}$ where $\varepsilon_{X}: X \rightarrow X \cup_{Z} Y$ and $\varepsilon_{Y}: Y \rightarrow X \cup_{Z} Y$ are the canonical quotient map $X \sqcup Y \rightarrow X \cup_{Z} Y$ restricted to $X$ and $Y$ respectively. 
We remark that more abstractly, one can get the pushout $X \cup_{Z} Y$ from the commutative $\mathrm{C}^{*}$-algebra $C(X) \oplus_{C(Z)} C(Y)$ which is the pullback of the pair $f^{*}: C(X) \rightarrow C(Z)$ and $g^{*}: C(Y) \rightarrow C(Z)$.

Proposition 4. The functor $\mathcal{B}^{\circledast-}$ preserves pushout in the sense that for any pushout $X \cup_{Z} Y$ given by $Z \stackrel{f}{\rightarrow} X$ and $Z \stackrel{g}{\rightarrow} Y$, the relaxed $C^{*}$-algebra $\left(\mathcal{B}^{\circledast\left(X \cup_{Z} Y\right)}, \mathfrak{T}_{\mathcal{B}^{\circledast}\left(X \cup_{Z} Y\right)}\right)$ satisfies: if $\left(\mathcal{B}^{\circledast X}, \mathfrak{T}_{\mathcal{B}^{\circledast X} X}\right) \stackrel{F}{\rightarrow}\left(\mathcal{C}, \mathfrak{T}_{\mathcal{C}}\right)$ and $\left(\mathcal{B}^{\circledast} Y, \mathfrak{T}_{\mathcal{B} \circledast Y}\right) \stackrel{G}{\rightarrow}\left(\mathcal{C}, \mathfrak{T}_{\mathcal{C}}\right)$ are relaxed ${ }^{*}$-homomorphisms such that $F \circ \mathcal{B}^{\circledast f}=G \circ \mathcal{B}^{\circledast g}$, then there is a unique relaxed ${ }^{*}$-homomorphism

$$
H:\left(\mathcal{B}^{\circledast\left(X \cup_{Z} Y\right)}, \mathfrak{T}_{\left.\mathcal{B}^{\circledast(X} \cup_{Z} Y\right)}\right) \rightarrow\left(\mathcal{C}, \mathfrak{T}_{\mathcal{C}}\right)
$$

such that $F=H \circ \mathcal{B}^{\circledast \varepsilon_{X}}$ and $G=H \circ \mathcal{B}^{\circledast \varepsilon_{Y}}$ for the canonical maps $\varepsilon_{X}: X \rightarrow X \cup_{Z} Y$ and $\varepsilon_{Y}: Y \rightarrow X \cup_{Z} Y$.

Proof. Note that $F$ and $G$ give rise to two well-defined *-homomorphisms

$$
\operatorname{id} \otimes F: C(X) \otimes\left(\mathcal{B}^{\circledast X}, \mathfrak{T}_{\mathcal{B} \circledast X}\right) \rightarrow C(X) \otimes\left(\mathcal{C}, \mathfrak{T}_{\mathcal{C}}\right)
$$

and

$$
\operatorname{id} \otimes G: C(Y) \otimes\left(\mathcal{B}^{\circledast Y}, \mathfrak{T}_{\mathcal{B}^{\circledast Y}}\right) \rightarrow C(Y) \otimes\left(\mathcal{C}, \mathfrak{T}_{\mathcal{C}}\right)
$$

respectively. Composing them with the canonical ${ }^{*}$-homomorphisms $\alpha_{X, \mathcal{B}}: \mathcal{B} \rightarrow C(X) \otimes$ $\left(\mathcal{B}^{\circledast X}, \mathfrak{T}_{\mathcal{B} \circledast X}\right)$ and $\alpha_{Y, \mathcal{B}}: \mathcal{B} \rightarrow C(Y) \otimes\left(\mathcal{B}^{\circledast Y}, \mathfrak{T}_{\mathcal{B} \circledast Y}\right)$ respectively, we get two ${ }^{*}$-homomorphisms $\phi: \mathcal{B} \rightarrow C(X) \otimes\left(\mathcal{C}, \mathfrak{T}_{\mathcal{C}}\right)$ and $\gamma: \mathcal{B} \rightarrow C(Y) \otimes\left(\mathcal{C}, \mathfrak{T}_{\mathcal{C}}\right)$. With

$$
(\phi(b))(x)=F\left(\alpha_{X, \mathcal{B}}(b)(x)\right)=F\left(\bar{\iota}_{x, \mathcal{B}}(b)\right)
$$

and

$$
(\gamma(b))(y)=G\left(\alpha_{Y, \mathcal{B}}(b)(y)\right)=G\left(\bar{\iota}_{y, \mathcal{B}}(b)\right)
$$

for all $(x, y) \in X \times Y$, we get

$$
\begin{aligned}
(\phi(b))(f(z)) & =F\left(\bar{\iota}_{f(z), \mathcal{B}}(b)\right)=F\left(\mathcal{B}^{\circledast f}\left(\bar{\iota}_{z, \mathcal{B}}(b)\right)\right) \\
& =G\left(\mathcal{B}^{\circledast g}\left(\bar{\iota}_{z, \mathcal{B}}(b)\right)\right)=G\left(\bar{\iota}_{g(z), \mathcal{B}}(b)\right)=(\gamma(b))(g(z))
\end{aligned}
$$

for all $z \in Z$. Thus $\phi(b) \in C(X) \otimes\left(\mathcal{C}, \mathfrak{T}_{\mathcal{C}}\right)$ and $\gamma(b) \in C(Y) \otimes\left(\mathcal{C}, \mathfrak{T}_{\mathcal{C}}\right)$ can be merged together to well define an element $\psi(b) \in C\left(X \cup_{Z} Y\right) \otimes\left(\mathcal{C}, \mathfrak{T}_{\mathcal{C}}\right)$ such that $(\psi(b))\left(\varepsilon_{X}(x)\right)=$ $(\phi(b))(x)$ and $(\psi(b))\left(\varepsilon_{Y}(y)\right)=(\gamma(b))(y)$ for all $(x, y) \in X \times Y$, and $\psi: \mathcal{B} \rightarrow C\left(X \cup_{Z} Y\right) \otimes$ $\left(\mathcal{C}, \mathfrak{T}_{\mathcal{C}}\right)$ is a well-defined ${ }^{*}$-homomorphism.

Then by Theorem 2 there exists a unique relaxed $*$-homomorphism

$$
\bar{\psi}:\left(\mathcal{B}^{\circledast\left(X \cup_{Z} Y\right)}, \mathfrak{T}_{\mathcal{B}^{\circledast}\left(X \cup_{Z} Y\right)}\right) \rightarrow\left(\mathcal{C}, \mathfrak{T}_{\mathcal{C}}\right)
$$

such that

$$
\bar{\psi}\left(\bar{\iota}_{p, \mathcal{B}}(b)\right)=(\psi(b))(p) \equiv \begin{cases}(\phi(b))(x)=F\left(\bar{\iota}_{x, \mathcal{B}}(b)\right), & \text { if } p=\varepsilon_{X}(x) \\ (\gamma(b))(y)=G\left(\bar{\iota}_{y, \mathcal{B}}(b)\right), & \text { if } p=\varepsilon_{Y}(y)\end{cases}
$$

for all $p \in X \cup_{Z} Y$ and $b \in \mathcal{B}$. Now with

$$
\bar{\iota}_{p, \mathcal{B}}(b) \equiv \begin{cases}\bar{\iota}_{\varepsilon_{X}(x), \mathcal{B}}(b) \equiv \mathcal{B}^{\circledast \varepsilon_{X}}\left(\bar{\iota}_{x, \mathcal{B}}(b)\right), & \text { if } p=\varepsilon_{X}(x) \\ \bar{\iota}_{\varepsilon_{Y}(y), \mathcal{B}}(b) \equiv \mathcal{B}^{\circledast \varepsilon_{Y}}\left(\bar{\iota}_{y, \mathcal{B}}(b)\right), & \text { if } p=\varepsilon_{Y}(y),\end{cases}
$$

the equality $(*)$ translates exactly to the equalities $F=\bar{\psi} \circ \mathcal{B}^{\circledast \varepsilon_{X}}$ and $G=\bar{\psi} \circ \mathcal{B}^{\circledast \varepsilon_{Y}}$, and so we simply take $H:=\bar{\psi}$. 


\section{References}

[1] D. Avitzour, Free products of $C^{*}$-algebras, Trans. Amer. Math. Soc. 271 (1982), 423-435.

[2] S. C. Kang, Quantum families of maps, Thesis, Univ. of Kansas, Lawrence, 2017.

[3] S. MacLane, Categories for the Working Mathematician, Grad. Texts in Math. 5, Springer, New York, 1971.

[4] G. K. Pedersen, Pullback and pushout constructions in $C^{*}$-algebra theory, J. Funct. Anal. 167 (1999), 243-344.

[5] P. M. Sołtan, Quantum families of maps and quantum semigroups on finite quantum spaces, J. Geom. Phys. 59 (2009), 354-368.

[6] P. M. Sołtan, On quantum semigroup actions on finite quantum spaces, Infin. Dimens. Anal. Quantum Probab. Relat. Top. 12 (2009), 503-509.

[7] P. M. Sołtan, On quantum maps into quantum semigroups, Houston J. Math. 40 (2014), 779-790.

[8] S. Wang, Free products of compact quantum groups, Comm. Math. Phys. 167 (1995), 671-692. 